YÁÑEZ RoMo, V. Y MUÑoz PARRA C., «Metodología para determinar la vulnerabilidad socio-territorial frente a la pobreza», REDUR I6, diciembre 20I8, págs. 95-120. ISSN I695-078X. doi: ı.18172/redur.4236

\title{
METODOLOGÍA PARA DETERMINAR LA VULNERABILIDAD SOCIO-TERRITORIAL FRENTE A LA POBREZA
}

\author{
Verónica YÁÑEZ ROMO \\ M.PHil. PlanificACIÓn RegionAl. Alemania \\ INGENIERA CIVIL \\ UNIVERSIDAD DE SANTIAGO DE CHILE
}

Carlos MUÑoz PARRA

ARQUITECTO

UNIVERSIDAD DE SANTIAGO DE CHILE ${ }^{\mathrm{T}}$

SumArio: I. Introducción. II. MARCo teórico. III. MEtodología. IV. Concepto de VULNERABILIDAD SOCIAL TERRITORIAL. V. ESTUDIO DE VARIABlES. VI. PONDERACIÓN DE SUBDIMENSIONES Y VARIABLES. VII. RESULTADOS. VIII. CONCLUSIÓN. IX. BIBLIOGRAFÍA.

RESUMEN: En conjunto con la Fundación Superación de la Pobreza y en agradecimiento al proyecto de Investigación DICYT N o9I3I2YR de la Universidad de Santiago (USACH), se trabaja sobre el concepto de vulnerabilidad social ${ }^{\mathrm{I}}$ relacionada estrechamente con los territorios urbanos y las problemáticas que estos presentan. Una vez definido el concepto de Vulnerabilidad Social Territorial se zonifica el territorio, en Macro-zonas de estudio y, según su división político administrativa, con el fin de identificar factores y variables determinantes que servirán de insumo para generar la propuesta metodológica que permita diagnosticar e identificar los diferentes niveles de vulnerabilidad social a escala regional y comunal, considerando los efectos territoriales. Esto ayudaría de forma más eficiente que las metodologías actuales a la focalización y mejoras de políticas, planes y/o programas del Gobierno, que vayan efectivamente dirigidas a los sectores de mayor rezago y escasos recursos.

PALABRAS ClAVE: vulnerabilidad socio-territorial; planificación territorial; ordenamiento territorial; superación de la pobreza; geomática aplicada, desarrollo sostenible.

\footnotetext{
' NOTA DE LA REVISTA: Este trabajo ha sido galardonado con el Premio Internacional de Investigación Interdisciplinar JUST-SIDE en su primera edición correspondiente al año 20I8. JUST-SIDE, acrónimo de «Justicia y Sostenibilidad en el Territorio a través de Infraestructuras de Datos Espaciales», es una Red Temática financiada por el Programa Iberoamericano de Ciencia y Tecnología para el Desarrollo (CYTED). Coordinada desde la Universidad de Coimbra, agrupa investigadores y empresas tecnológicas de diferentes campos que confluyen en el ámbito del Geoderecho de Argentina, Brasil, Costa Rica, Cuba, Chile, España, México, Portugal y Uruguay. La aportación jurídica desde España está confiada al Área de Derecho Administrativo de la Universidad de La Rioja.
} 


\section{METHODOLOGY TO DETERMINE SOCIO-TERRITORIAL VULNERABILITY TO POVERTY}

ABSTRACT: Together with the Foundation for Overcoming Poverty and with appreciation to the Research Project No. 09I3I2YR DICYT at the University of Santiago, working on the concept of Social vulnerability closely related to urban areas and the problems they present. Once defined the concept of Territorial Social Vulnerability, territory is zoned in Macro- study areas and by administrative and political divisions in order to identify determining factors and variables that serve as input to generate the methodological proposal for diagnosis and identify the different levels of social vulnerability to regional and municipal level, considering territory effects. This would help more efficiently to the current methods of targeting and improved policies, plans and / or government programs that are aimed at the more vulnerable and poor population.

KEYWORDS: socio-territorial vulnerability; territorial planning; territorial order; overcoming of poverty; applied geomatics; sustainable development.

\section{Introducción}

En Chile las actuales políticas públicas del ámbito social dependen no solo del rol del estado sino también de una sociedad civil más empoderada y de un mercado versátil y globalizado.

Esto ha provocado un conjunto de visiones, intereses y experiencias sectoriales, que con distinta intensidad y focalización, están reconfigurando el cómo la sociedad chilena promueve el desarrollo social y orienta su acción hacia la superación de la pobreza (CEPAL, I997; PNUD 2000). En este sentido, se observa que existe una carencia de políticas sociales efectivas que den respuesta a las actuales demandas de la sociedad; la efectividad e impacto de los programas sociales están por debajo de su potencialidad y la inserción de criterios y recursos privados en el diseño de planes y programas aún no responde a una acción coordinada, generando un escenario autorreferente y fragmentado desde la perspectiva de los grupos sociales en situación de pobreza (Gacitúa, Sojo y Shelton, 2000).

Un claro ejemplo de ello en relación a las políticas de vivienda y territorio es el mismo Ministerio de Vivienda y Urbanismo quien declara que «El Estado, ha generado diversas políticas y líneas de intervención para enfrentar el problema de la pobreza, siendo la producción de viviendas y determinados equipamientos lo más significativo en lo urbano, en consideración al alto déficit heredado. No obstante, lo que ha caracterizado la intervención estatal es que ha operado fundamentalmente desde lo sectorial con escaso énfasis en coordinar y focalizar políticas con una lógica territorial». Las actuaciones sectoriales sólo han conseguido aliviar alguna dimensión del problema de la pobreza (vivienda social, empleo de emergencia, subsidios, etc.), pero no han logrado configurar oportunidades sustentables (Ministerio de Vivienda y Urbanismo, 2006).

A modo de configurar el perfil de la temática del Hábitat Vulnerable, que denominaremos como Hábitat Residencial con Altas Restricciones, señalaremos que constituye una forma de intervención humana en el contexto territorial donde se adecuan espacios de manera directa, paulatina y fundada en visiones de organización política, económica y cultural, (Caro, 2003). Esto en el afán humano de atender los requerimientos cotidianos básicos de vida (ser, estar, tener) y las interrelaciones con sus semejantes, lo cual es producto de una intervención humana factible, dentro de ciertos parámetros de 
posibilidades que le otorga el contexto territorial. Es aquí entonces donde surge el tema de las restricciones, cuando se toca el aspecto de lo posible por la diversidad de sus componentes, y se considera la administración de la gestión de los recursos disponibles incluyendo la innovación como adaptación en función de la conservación (Vignolo, 2004). La clave para adaptarse al cambio del entorno, manteniendo o incrementando el bienestar, está en lo que se conserva, no en lo que se innova, de esta manera se genera resiliencia contrarrestando la vulnerabilidad.

La vulnerabilidad es la pérdida esperable de un determinado bien expuesto, puede tratarse de vulnerabilidad humana, estructural, económica o ecológica, de acuerdo con el tipo de riesgo a evaluar, es necesario tener una aproximación para saber que se requiere conservar y que se precisa innovar, dentro del proceso de generación de un hábitat pertinente con las necesidades y aspiraciones de un demandante específico. Pues este proviene de distintos contextos territoriales en los cuales está insertado y de donde adquiere sus particularidades morfológicas, funcionales y simbólicas. Todo esto con una amplia diversidad de sus componentes, tanto los de carácter físico-espacial como lo climatológico, geográfico y topográfico como los de carácter sociocultural, entendiendo por ello los sentimientos de identificación, de arraigo, de vínculos sociales y rescate de sus valores culturales. De esta manera, usamos la noción de «Vulnerabilidad Social Territorial», a partir de que resulta fundamental instalar una perspectiva territorial en las decisiones con la finalidad de superar la parcialidad sectorial de ellas y producir cambios en la situación de vulnerabilidad de la población y una integración territorial y sectorial efectiva.

El Gobierno de Chile desea fortalecer la articulación del sistema de protección social a nivel regional y local, para esto es necesario identificar de forma integral la localización de los pobladores más vulnerables social, ambiental y económicamente, considerando los efectos propios de cada territorio sobre cada uno de éstos. Para dar respuesta a esta necesidad el Observatorio Social perteneciente al Ministerio de Desarrollo Social, solicitó al Departamento de Ingeniería Geográfica de la Universidad de Santiago de Chile orientación académica para generar una definición propia con respecto al término de «Vulnerabilidad Social Territorial», y además proponer una nueva metodología que permitiese integrar la variable territorial con sus diversas unidades o ámbitos geográficos a escala nacional, regional y comunal.

Finalmente con este proyecto de investigación nos propusimos realizar un estudio integrador considerando el ámbito científico y las ciencias sociales, que serán la base para generar una propuesta metodológica basada en análisis cuantitativo y cualitativo. En este sentido, nuestro análisis se centró en el estudio de la vulnerabilidad social incorporando la variable territorial e integrando los aspectos sociales, económicos, ambientales, culturales y normativos. Esta propuesta metodológica, se probó empíricamente en la Región de Antofagasta y se probó en la Región de O’Higgins, apoyada por una red de investigadores pertenecientes a la Fundación Superación de la Pobreza. 


\section{Marco teórico}

El término vulnerabilidad puede aplicarse a una persona o a un grupo social según su capacidad para prevenir, resistir y sobreponerse de un impacto. Las personas vulnerables son aquellas que, por distintos motivos, no tienen desarrollada esta capacidad y que, por lo tanto, se encuentran en situación de riesgo. El concepto de «vulnerabilidad» tiene sus cimientos en las diferencias socioeconómicas que se dan en la sociedad, comprendiendo que se refiere a desventajas o riesgos que la movilización de los activos que disponen las personas, hogares, comunidades y territorios no permiten aprovechar oportunidades de integración y movilidad social o contrarrestar riesgos de pérdida de bienestar, (Arriagada, 200I). Para otro autor (Kaztman, 2003) la utilidad de los activos depende de su ajuste a las exigencias de la estructura de oportunidades vigentes.

El concepto de la vulnerabilidad nace de las ciencias humanas y sociológicas, el que fue adoptado y enriquecido por expertos en desarrollo y pobreza. En sus primeras aproximaciones este término se vincula desde el punto de vista social con situaciones de indefensión, temor e inseguridad que las personas viven, relacionados a problemas de desocupación, bajos ingresos y dificultades en el acceso a servicios básicos. En Europa a partir de los años 90 este concepto se utiliza en gran parte a la toma de conciencia colectiva de la amenaza que pesa sobre segmentos cada vez más numerosos y mal protegidos de la población, el debate sitúa los términos del problema no en el crecimiento económico, ni en la producción de riquezas, sino en la manera de repartirla y de tener acceso a ella. «El dualismo social se plantea a partir de la creciente división social entre los que participan de los beneficios de la modernidad, gozando de ingresos suficientes y estables, y aquellos que excluidos de los beneficios de la modernidad, viven con ingresos insuficientes y trabajan en situación precaria», (CEPAL, 2006).

En Latinoamérica el concepto de Vulnerabilidad inicialmente se relaciona a definiciones de Vulnerabilidad Social con enfoques de carencia de poder y privación de derechos humanos (Bustamante, 2000), otros en relación a pobreza y niveles socioeconómicos, y para algunos autores (Pizarro, 200I) la vulnerabilidad social explica la incapacidad de los grupos más débiles de la sociedad para enfrentar el modelo económico neoliberal vigente.

Actualmente los países latinoamericanos están atravesando intensas transformaciones, en el marco de los procesos de globalización, con reestructuración económica que comprende reformas del Estado, apertura del mercado financiero, incremento de la tercerización entre otros. Junto a esto cambia el tipo de desarrollo social; hay aceptación de la desigualdad y «la noción de igualdad ha sido reemplazada por la de equidad (que es una parte de la igualdad), la agenda social se ha fraccionado y se ha ampliado para contemplar temas como la extrema pobreza, la equidad de género, de raza y de etnia, entre otros», (CEPAL, 2006). Es así que en Latinoamérica también se incorpora y estudia el concepto de Manejo Social de Riesgo, cuyo enfoque nace en la década del 90 en el Banco Mundial, entendiéndose por riesgo la vulnerabilidad que las personas, las comunidades y los territorios tienen para enfrentar situaciones que pueden afectarlos negativamente. Este tipo de vulnerabilidad se refiere a la probabilidad de resultar perjudicados por la ocurrencia de sucesos inesperados provocados por los desastres 
naturales, ambientales o ecológicos. Es así que en este contexto algunos autores (Zapata, 2000, extraído de CEPAL, 20I0) definen la vulnerabilidad como «la probabilidad de que debido a la intensidad de un evento externo y a la fragilidad de los elementos expuestos ocurran daños en la economía, la vida humana y el ambiente».

En el caso del Estado Chileno, se ha tratado el concepto de vulnerabilidad bajo la perspectiva socioeconómica, desarrollando la Estrategia de Protección Social, la cual se orienta principalmente en el bienestar de las personas; garantizando derechos que permiten llevar una vida digna e integrada en el entorno social, cultural y económico. Esto implica un fuerte énfasis preventivo e identificativo de lugares donde la población puede o está en situaciones de carencias de todo tipo que impidan su bienestar, teniendo como desafío fortalecer la articulación del Sistema de Protección Social en los niveles regionales y locales. Se incorpora explícitamente al «territorio» como elemento estratégico clave para el diseño y ejecución de las políticas sociales en el entendido que es allí donde se desarrolla la vida cotidiana de las personas.

Esto hace necesario recopilar y revisar las definiciones sobre el concepto de «vulnerabilidad social territorial» con el fin de escoger o crear una propia para generar una nueva herramienta metodológica integral que permita identificar y caracterizar los tipos de vulnerabilidad (social, económica, ambiental) que pueden estar presentes en un determinado territorio con el fin de mejorar la futura localización de los pobladores y su calidad de vida como también mejorar el impacto de las políticas sociales, al evaluar y controlar los programas sociales ya existentes por parte del Ministerio de Desarrollo Social en Chile.

\section{Metodología}

Para la realización del diagnóstico y la determinación del instrumento que permite identificar los niveles de vulnerabilidad social-territorial de la región, primeramente se comienza con una revisión bibliográfica que da cuenta de los diferentes conceptos y definiciones que abarca este término. En este caso se establece la definición del concepto de Vulnerabilidad Social Territorial según el concepto construido por el Ministerio de Desarrollo Social MDS (ex MIDE-PLAN) y el German Technical Cooperation Agency GTZ. El móvil de emplearlo se sustenta en dos motivos: su robusta construcción y la finalidad de comparar ambas metodologías, entendiéndose por Vulnerabilidad Social Territorial como «La incapacidad de impedir que acontecimientos de diversa índole afecten negativamente las condiciones de vida de la población que habita un territorio determinado, sea por falta o insuficiencia de activos protectores de riesgos como por la falta de condiciones para aprovechar el flujo de oportunidades».

Esta mirada comprende al Territorio como una «construcción social» que trasciende los aspectos físicos-geográficos que constituyen su base, incorporando además muy centralmente a la población y a las comunidades, junto a elementos de intervención humana que se «instalan» en él, como por ejemplo la presencia de caminos, acueductos , viviendas, escuelas, hospitales, cárceles, comisarías y cuarteles policiales. 
La mención de los «activos» se refiere al stock o conjunto de recursos de diversos tipos, que posee la población, hogares y comunidades de un territorio, sobre los que se tiene control y cuya tenencia y utilización permite evitar el deterioro de las condiciones de vida o disminuir los riesgos ante la ocurrencia de un evento inesperado o de resultados inciertos. «La vulnerabilidad supone una variabilidad en el tiempo que sintoniza con las variaciones de los activos» (Busso, 200I).

En contraposición, el término de «oportunidades», se refiere a un flujo de eventos y sucesos que constituyen verdaderas ventanas de capacidades de crecimiento e integración para la población, hogares y/o comunidades territoriales. Conceptualmente se habla de oportunidades y no de activos por ser cambios presentes en el espacio físico, económico y/o social territorialmente provenientes de elementos propios de la intervención humana. Cuando la población hace uso de las oportunidades, el conjunto de activos que posee aumenta o mejora en cuanto a su calidad.

En función de las distintas dimensiones que componen el término y en base a bibliografía relacionada, se fijan los lineamientos y directrices del estudio. Tras la definición del concepto se genera el desarrollo de la propuesta metodológica de carácter cuantitativo y cualitativo, la zonificación del territorio se realiza en macro-zonas de estudio Se trabaja con las regiones propuestas por la Corporación de Fomento - CORFO (Geografía económica de Chile, I950), donde se divide al territorio en seis (6) grandes zonas, considerando características físicas, humanas y económicas y además según su división político administrativa, se trabaja a escala regional y comunal con el fin de identificar factores y variables determinantes. Cabe señalar que se optó por la regionalización de la CORFO pues presenta similitudes a la propuesta Proyecto País del Colegio de Ingenieros de Chile. También se debe considerar que Chile es un país diverso en su geografía de Norte a Sur que presenta fuertes contrastes, por esta razón, trabajar con Macrozonas que presentan características ambientales y territoriales similares permite una mejor selección de las variables físicas, humanas y económicas lo que ayuda a diagnosticar mejor el área de estudio.

A continuación, se detalla el proceso investigativo emprendido en la construcción de la propuesta metodológica (figura I). En primer lugar se presenta un diagrama para luego detallar los pasos emprendidos. 


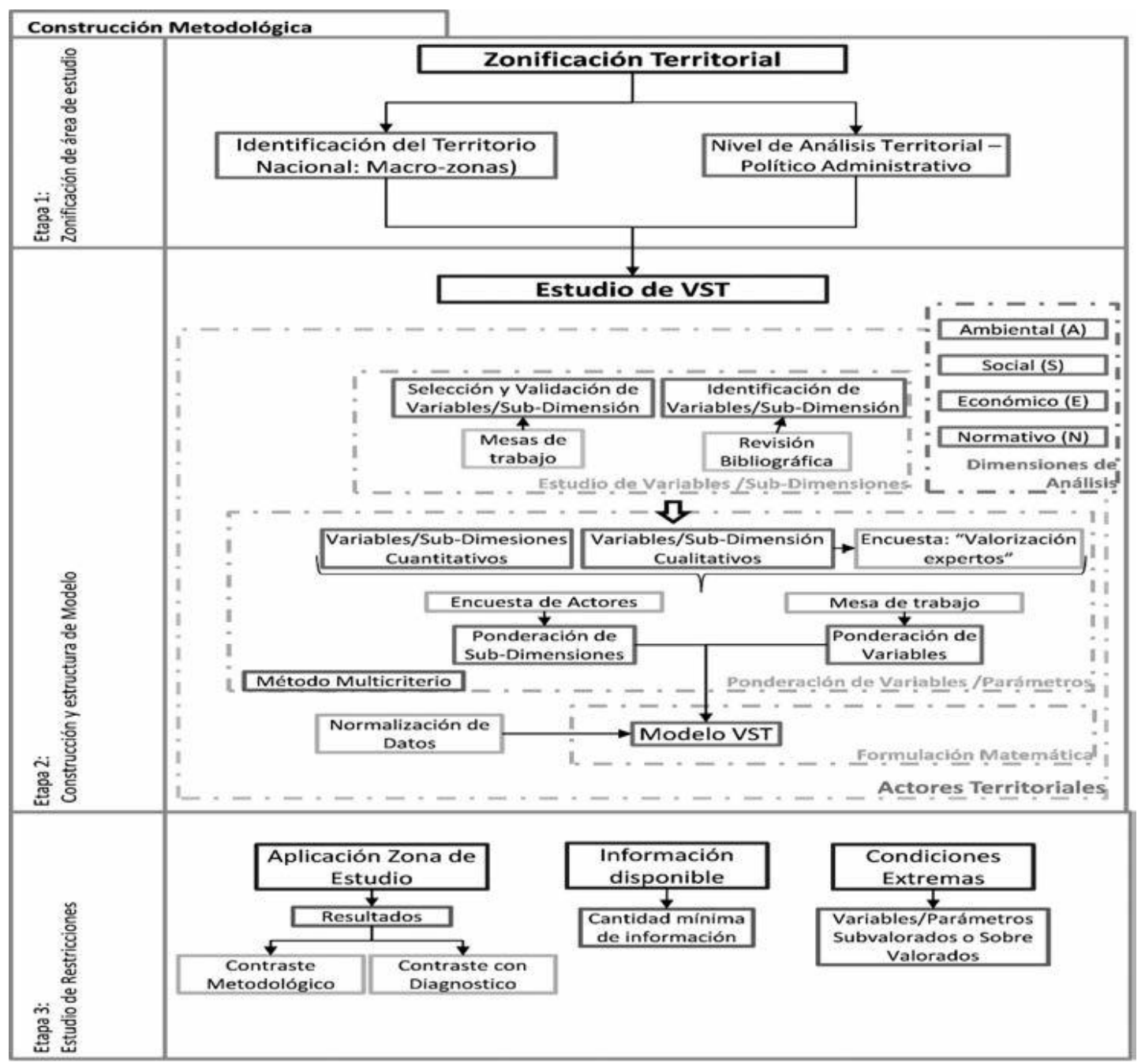

Figura I. Esquema de metodología de investigación. Fuente: Equipo de investigación del área de Ordenamiento Territorial, Departamento de Ingeniería Geográfica, Universidad de Santiago de Chile, año 20I3.

\section{Concepto de vulnerabilidad social territorial}

Para los efectos de este estudio se entenderá por vulnerabilidad social territorial «la incapacidad de impedir que acontecimientos de diversa índole afecten negativamente las condiciones de vida de la población que habita un territorio determinado, sea por falta o insuficiencia de activos protectores de riesgos como por la falta de condiciones para aprovechar el flujo de oportunidades».

Los conceptos claves empleados en esta definición son los siguientes:

- Territorio: Es una 'construcción social' que trasciende los aspectos físicosgeográficos que constituyen su base, sino que además incorpora muy centralmente a la población y comunidades, junto a elementos de intervención humana que se 'instalan' en él, como por ejemplo, la presencia de caminos, acueductos , viviendas, escuelas, hospitales, cárceles, comisarías y cuarteles policiales.

- Activos: Se refieren al stock o conjunto de recursos de diversos tipos, que posee la población, hogares y comunidades de un territorio, sobre los que se tiene control y cuya tenencia y utilización permite evitar el deterioro de las condiciones de vida o 
disminuís los riesgos ante la ocurrencia de un evento inesperado o de resultados inciertos. Se trata de aquellos recursos con que cuenta la población a partir del hecho de habitar un territorio determinado. Dicho de otra manera, los Activos configuran un stock acumulado a lo largo del tiempo por la acción del estado, mercado ciudadanía con el que se cuenta para reaccionar ante eventos específicos. La potencialidad del concepto de Activos radica en introducir distinciones fundamentales de las situaciones de desventaja social vislumbrando intervenciones diferenciadas en función del stock de activos y que significarán un flujo o acción de modificación del nivel, diversidad, grado de fortaleza o flexibilidad de los recursos. La vulnerabilidad supone una variabilidad en el tiempo que sintoniza con las variaciones de los activos (Busso, 200I: 24).

- Oportunidades: Se refieren a un flujo de eventos y sucesos que constituyen verdaderas ventanas de oportunidades de crecimiento e integración para la población, hogares y/o comunidades territoriales. Conceptualmente se habla de oportunidades y no de activos por ser cambios presentes en el espacio físico, económico y/o social territorial provenientes de elementos propios de la intervención humana. Dice relación más específicamente, a mejoras que la sociedad le ofrece a la población para su uso y que tendrá como resultado un mayor bienestar. En definitiva, las Oportunidades deben ser vistas como una ventana disponible que ayuda a aumentar o mejorar el stock de activos. Cuando la población hace uso de las oportunidades, el conjunto de activos que posee au-menta o mejora en cuanto a su calidad.

- Zonificación Territorial: Con objeto de aplicar de forma consistente el concepto enunciado de Vulnerabilidad Social Territorial, y comprendiendo que el espacio geográfico de Chile posee una gama de contrastes a largo y ancho del territorio, se torna necesario realizar una revisión bibliográfica de cómo se ha limitado los territorios durante la historia del país. De la información obtenida, se puede destacar dos tipos de divisiones del espacio geográfico: Macro-zonificación y Política-Administrativa, las cuales se detallan a continuación:

- Identificación de Macrozonas: Se identifica el área de estudio en función de la Macrozonas. Se trabaja con las regiones propuestas por la CORFO, en donde se divide al territorio en seis (6) grandes zonas, Norte Grande, Norte Chico, Zona Central, Concepción y la Frontera, Región de Los Lagos, Región de los Canales.

- Regiones Político-Administrativa: Las regiones político-administrativas de Chile son las divisiones territoriales superiores, las cuales se subdividen en provincias y éstas a su vez en comunas. Desde 1974, Chile ha estado políticamente dividido en regiones administrativas. Hasta 2007, las regiones de Chile fueron I3; en la actualidad, el total es de I5 regiones, incluyendo las regiones de Arica y Parinacota, y Los Rios.

La Regionalización se basa en la división político-administrativa dado que en ellas se sustentan las decisiones políticas (aplicación de políticas públicas). Por lo estudiado en los puntos anteriores, y por los puntos de vista de análisis 
mencionado (económico, social, ambiental y normativo), es conveniente focalizar el análisis en el área de estudio de la siguiente forma:

- Primero, seleccionar una macrozona de estudio, puesto que su agrupación se basa en tópicos comunes de cada área (física, humana y económica) y

- Luego, seleccionar el nivel político-administrativo para aplicar el instrumento, desde; nivel general de análisis (región), hasta las unidades territoriales en las cuales se aplicará en detalle (comuna).

- Actores Territoriales: Por la naturaleza inclusiva que se pretende dar a este estudio, se torna necesario incorporar a los siguientes actores:

- Actores Públicos Privados

- Sociedad Civil

Los procesos en los cuales se encuentran involucrados los actores son los siguientes:

Tabla I. Tipos de actores considerados. Fuente: Equipo de investigación del área de Ordenamiento Territorial, Departamento de Ingeniería Geográfica, Universidad de Santiago, año 20I3.

\begin{tabular}{l|l}
\hline \multicolumn{1}{c|}{ Procesos } & \multicolumn{1}{c}{ Actores } \\
\hline Validación de Variables & $\begin{array}{l}\text { Grupo de expertos - Mesas de trabajo con Fundación } \\
\text { Superación de la Pobreza }\end{array}$ \\
\hline Ponderación de Sub- & $\begin{array}{l}\text { Todos los actores territoriales de la Zona de Estudio, } \\
\text { Instituciones Públicas y expertos de la red de Fundación } \\
\text { Superación de la Pobreza }\end{array}$ \\
\hline Dimensiones & Expertos Territoriales - Zona de Estudio \\
\hline Vonderación de Variables & Expertos Territoriales - Zona de Estudio \\
\hline
\end{tabular}

\section{Estudio de variables}

Las variables consideradas son productos de la revisión bibliográfica detallada en bibliografía. Su validación se realizó en mesas de trabajo con expertos en donde la Fundación Superación de la Pobreza nos apoyó con una red de expertos que trabajan en instituciones públicas como privadas, con la finalidad de revisarlas y discutirlas, modificando, eliminando e incorporando nuevas variables. Se logró así un conjunto de variables finales producto de los dos pasos anteriores. A continuación se detalla el trabajo emprendido en cada etapa.

\section{Etapa I: Variables Consideradas}

Durante el proceso de revisión bibliográfica se identificaron diferentes variables, las cuales se agruparon en sub-dimensiones que están relacionadas con las diferentes dimensiones de análisis abordadas, lo social, económico, ambiental y normativo. En la figura 2 se observan las sub-dimensiones en las cuáles se agruparon las diferentes variables. Posteriormente, se definen las variables abordadas en cada sub-dimensión. 


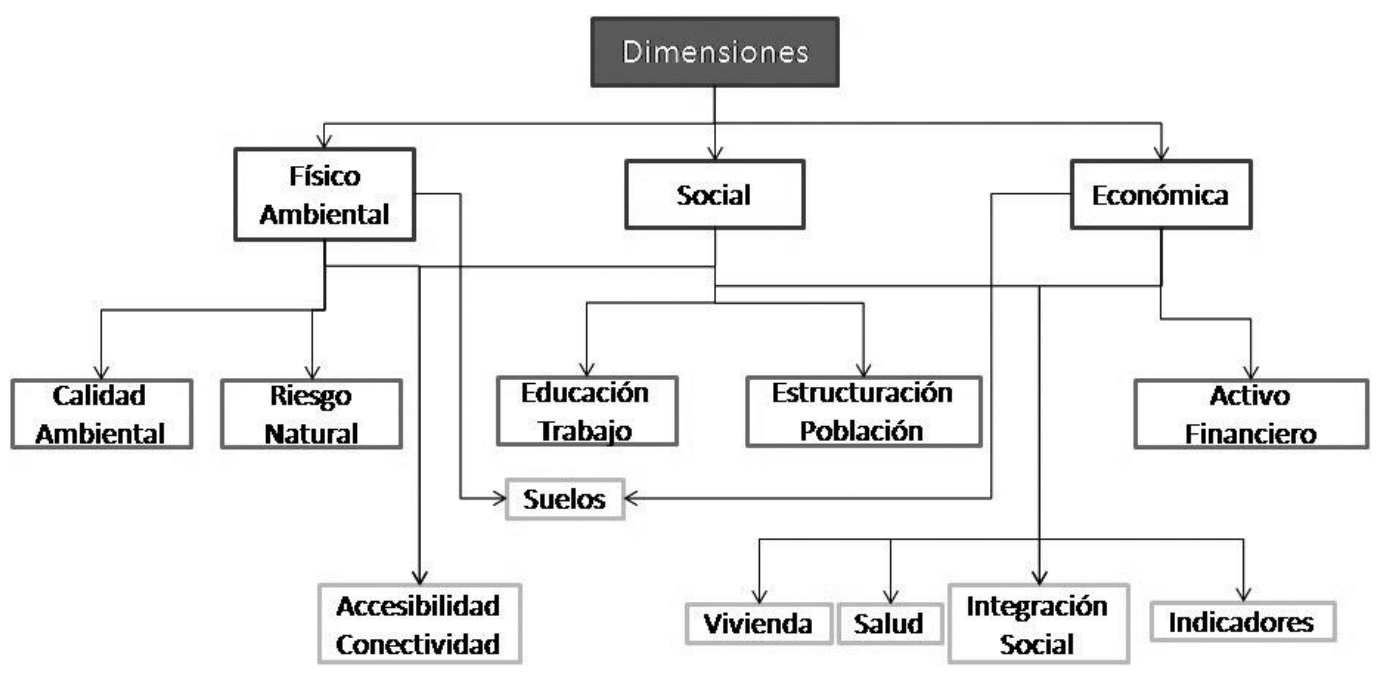

FIGURA 2. Esquema de variables seleccionadas. Fuente: Equipo de investigación del área de Ordenamiento Territorial, Departamento de Ingeniería Geográfica, Universidad de Santiago, año 2013 .

- Calidad del agua:

- Concentración de sólidos: El término concentración de sólidos se refiere a la proporción de sólidos minerales en suspensión en un determinado cuerpo de agua.

- Sustancias tóxicas: Bajo esta denominación se incluyen un conjunto de sustancias tales como desechos industriales, metales pesados y agroquímicas cuyo consumo puede generar reacciones de toxicidad leves o severas en la población humana y animal asentada en la cuenca. Las fuentes de sustancias tóxicas son las actividades industrial, minera y agrícola.

- Compuestos orgánicos, nutrientes y patógenos: La presencia en altas concentraciones de compuestos orgánicos tales como heces, sangre y restos de animales.

- Suelos:

- Valor Promedio del m2 de Terreno: Las características de un terreno inciden directamente en su valor (ubicación, ocupación, uso tipo de superficie y orientación), entre más elementos positivos mayor es su valor. De ésta forma, se restringe la adquisición de éstos.

- Contaminación del suelo: Se refiere a la presencia de restos de sustancias químicas provenientes de actividades antrópicas como, por ejemplo, agroquímicos y/o desechos.

- Capacidad de uso del suelo: El término «capacidad de uso del suelo» se refiere al potencial de un suelo como recurso para desarrollar diferentes cultivos y formas de agricultura.

- Relieve, topografía y paisaje: El término relieve se refiere a la configuración fisiográfica de una cuenca o de sectores dentro de ella. 
- Geodinámica:

- Inestabilidad de laderas: Es el proceso por el cual las laderas naturales o los taludes construidos por actividades antrópicas pierden estabili-dad y se deslizan a través de una superficie de ruptura.

- Fallas activas: El análisis de los rasgos tectónicos permite definir o determinar las fallas y las situaciones de riesgo sísmico que caracterizan las zonas de interés.

- Biota:

- Biodiversidad: El término biodiversidad o diversidad biológica alude a la variabilidad de organismos vivientes y hábitats, así como a los complejos ecológicos de los cuales forman parte. El término biodiversidad incluye la diversidad al interior de las especies, entre especies y de ecosistemas.

- Porcentaje de Superficie Protegida del Deterioro Ambiental: Área silvestres protegidas del Estado y Sitios Prioritarios para la Biodiversidad. El supuesto propuesto es: «A mayor cantidad de área protegidas, menor es el riesgo de deterioro ambiental».

- Educación / Trabajo:

- Escolaridad Promedio: número promedio de años cursados en el sistema educacional, desde primero básico en adelante. Se aplica a las personas de 15 años y más.

- Inacción Juvenil: «La inacción juvenil son el desempleo y retraso escolar, los cuales son fenómenos indesmentibles de la desintegración social».«Inacción es considerada precursor de la delincuencia y dro-gadicción» (Sabatini, Cáceres y Cerda).

- Vivienda:

- Ingresos del Jefe de Hogar o del grupo familiar: son los ingresos por concepto de sueldos y salarios, ganancias provenientes del trabajo independiente, incluido el auto suministro y el valor del consumo de productos agrícolas.

- Disponibilidad de agua potable: Se consideró como indicador el porcentaje de hogares de la comuna cuya disponibilidad de agua potable califica como aceptable de acuerdo a si posee llave dentro de la vivienda o no.

- Sistema de eliminación de excretas: El indicador considerado en este caso es el porcentaje de hogares de la comuna cuyo sistema de eliminación de excretas califica como aceptable de acuerdo si posee WC conectado a alcantarillado o fosa séptica (aceptable), deficitaria en otro caso.

- Materialidad de la vivienda: Este concepto se relaciona con los materiales predominantes en paredes exteriores, cubierta de techo y pisos. Considerado como un activo, asociado a las condiciones con las cuales los habitantes deben enfrentar diferentes acontecimientos (naturales, sociales).

- Saneamiento: Considera el sistema de distribución de agua potable al interior de la vivienda y el medio de eliminación de excretas, según las categorías de aceptable y deficitario.

- Hacinamiento: Según lo establecido por el Ministerio de Vivienda, los criterios de hacinamiento son los siguientes: 
- Sin hacinamiento: si hay 2,4 o menos personas por dormitorio.

- Con hacinamiento: si hay 2,5 o más personas por dormitorio. Favorece la transmisión de la pobreza. Dificulta la realización de tareas escolares, las relaciones familiares.

- Viviendas Irrecuperables: Se refiere a las viviendas que presentan grados de deterioro parcial o total. Una vivienda irrecuperable genera un desmejoramiento desde el punto de vista de habitabilidad y visual.

- Accesibilidad-Conectividad:

- Accesibilidad al Transporte: Referida al acceso a medios de transporte, así también como al grado de accesibilidad y conexión entre territorios.

- Accesibilidad a los Servicios de Salud: «La ausencia o dificultad en la accesibilidad es un elemento central en el deterioro urbano de las ciudades, en cuanto a la ausencia o insuficiencia en la infraestructura de servicios en general y públicos en particular, son los servicios de salud uno de los tópicos más sensibles dada la demanda existente por parte de la población».

- Accesibilidad a Establecimientos Educacionales: «Es un servicio esen-cial para alcanzar calidad de vida con estándares aceptables, de lo contrario en este caso particular, dificultades en la accesibilidad y presencia de deterioro puede conllevar niveles de marginalidad en la población» (Romero, 200I). Por lo tanto, la distancia y el tipo de establecimientos son esenciales.

- Accesos a Polos de Desarrollo: El acceso a los servicios de primera necesidad y de ocio es un elemento esencial para determinar el grado de aislamiento. El supuesto metodológico es que a mayor dificultad de acceso a los centros urbanos, que proveen una serie de servicios complementarios a los provistos por el Estado.

- Acceso a telecomunicaciones: Para la determinación de la integración en telecomunicaciones se opta por el indicador de líneas de Internet banda ancha por cantidad de habitantes, debido a que explica, sin sobrevalorar o subvalorar, como son los casos de antenas radioemisoras y de repetición de televisión abierta, que dependen de su localización en función de la cobertura que presentan.

- Estructura de Población:

- Envejecimiento de Población (porcentaje población tercera edad): Contribuye a generar dependencia demográfica.

- Habitabilidad del Territorio: Se refiere a las condiciones naturales (físico-ambientales) que presenta el territorio las cuales pueden facilitar o dificultar el habitarlo.

- Migración (interurbana e internacional): Se asocia a competencia laboral, aglomeración, marginalidad.

- Hacinamiento Territorial: Relacionada con las viviendas existentes en un territorio (Densidad) y la disposición de éstas.

- Integración Económica:

- Tasa de Detenciones según denuncias de Delitos de Mayor Connotación social (DMCS): Asociada a la percepción de la seguridad ciudadana. 
- Línea de Pobreza: Se determina por el nivel de ingreso mínimo necesario por persona para cubrir el costo de una canasta individual para la satisfacción de las necesidades alimentarias y no alimentarias.

- Línea de Indigencia: Corresponde al ingreso mínimo necesario por persona para cubrir el costo de una canasta alimentaria.

- Activo Financiero:

- Fondo Común Municipal (FCM) Autonomía: «Mientras más autonomía tenga una comuna del FCM, menor será su vulnerabilidad social territorial. Se entiende que la dependencia elevada del FCM significa debilidad del municipio y territorio para localizar actividades generadoras de ingresos propios: patentes, permisos de circulación y permisos de edificación de viviendas no exentas de contribuciones».

- Salud:

- Indicadores Nutricionales: Grado de desnutrición y/o sobrepeso que presenta la población infantil de un área.

- Infraestructura de Salud (hospitalaria): Tipo de recinto de salud.

- Enfermedades, Complejidad de Enfermedades: Complejidad de Enfermedades que presentan los habitantes del área de estudio (asociada a tratamientos, curso de la enfermedad, dependencia).

\section{Etapa II: Validación de Variables}

De acuerdo a lo planteado, fue necesaria la validación de las variables propuestas. Ésta se llevó a cabo por medio de dos mesas de trabajo. La primera fue integrada por profesionales de la Fundación Superación de la Pobreza y la segunda por profesionales de la Universidad de Santiago de Chile. En los siguientes puntos se especifica el trabajo realizado en cada una, finalizando con el análisis de ambas.

\section{Primera mesa de trabajo: Fundación Superación de la Pobreza (FSP)}

Esta mesa de trabajo fue realizada de manera virtual, en donde las instrucciones e ilustraciones de las variables propuestas fue mediante videoconferencia el día 02 de agosto de 2013 con Jennifer Smith, Jefe Territorial de Antofagasta FSP. La mesa contó con la presencia de profesionales de las siguientes especialidades: Medicina Veterinaria; Trabajo Social; Psicología; Kinesiología; Pedagogía de historia y geografía. El resultado de la mesa fue una serie de sugerencias y recomendaciones, las cuales son sintetizadas en el tabla 2.

TABLA 2. Validación de variables fundación. Fuente: Equipo de investigación del área de

Ordenamiento Territorial, Departamento de Ingeniería Geográfica, Universidad de Santiago, año 2013 .

\begin{tabular}{l|c|l}
\hline Variables Sugeridas & \multicolumn{2}{|l}{} \\
\hline Variables & Mesa de trabajo & Observación \\
\hline Integración Social & FSP & $\begin{array}{l}\text { Se refiere a: Asociatividad; Existencias de } \\
\text { organizaciones comunitarias; Gobiernos locales y } \\
\text { regionales; Vinculación con el sector privado }\end{array}$ \\
\hline
\end{tabular}




\begin{tabular}{l|c|l}
\hline $\begin{array}{l}\text { Dotación de personal } \\
\text { (Salud) }\end{array}$ & FSP & $\begin{array}{l}\text { Personal con el que cuentan los servicios de salud } \\
\text { (especialización) }\end{array}$ \\
\hline Población Indígena & FSP & $\begin{array}{l}\text { Identificación de población perteneciente a } \\
\text { etnias, siendo incluida en Ítem de estructura de } \\
\text { población. }\end{array}$ \\
\hline Recomendaciones & \multicolumn{2}{|l}{} \\
\hline Consideraciones & Mesa de trabajo & Observación \\
\hline Educación/trabajo & FSP & Separar Ítem \\
\hline SIMCE & FSP & Considerar resultados del SIMCE en el análisis \\
\hline Inacción Juvenil & FSP & $\begin{array}{l}\text { Reemplazar el concepto por “Empleabilidad } \\
\text { Juvenil” }\end{array}$ \\
\hline
\end{tabular}

2. Segunda mesa de trabajo: Universidad de Santiago de Chile (USACH)

Esta mesa de trabajo compuesta por profesionales de las siguientes especialidades: Arquitectura, Ingeniería Civil en Geografía, Ingeniería Ambiental, Psicología. En la mesa de trabajo se propuso una serie de sugerencias y recomendaciones, las cuales se detallan en el tabla 3.

TABLA 3. Validación de variables USACH. Fuente: Equipo de investigación del área de Ordenamiento Territorial, Departamento de Ingeniería Geográfica, Universidad de Santiago, año 2013.

\begin{tabular}{|c|c|c|}
\hline \multicolumn{3}{|l|}{ Variables Sugeridas } \\
\hline Variables & Mesa de trabajo & Observación \\
\hline Integración Social & USACH & Segmentación de la población \\
\hline \multicolumn{3}{|l|}{ Recomendaciones } \\
\hline Consideraciones & Mesa de trabajo & Observación \\
\hline Etnia & USACH & $\begin{array}{l}\text { Se sugirió la identificación de población } \\
\text { pertenecientes a Etnias }\end{array}$ \\
\hline \% áreas verdes & USACH & Incluir dentro del estudio las áreas verdes \\
\hline $\begin{array}{l}\text { Clasificación por tipo } \\
\text { de trabajo }\end{array}$ & USACH & $\begin{array}{l}\text { Identificar los tipos de trabajos que se desempeñan } \\
\text { en área de estudio }\end{array}$ \\
\hline Migración & USACH & $\begin{array}{l}\text { Diferenciar el tipo de migración, en: Estructura } \\
\text { (Interna-Externa); motivos de la migración } \\
\text { (estudios- trabajo); Internacional; Diferenciación } \\
\text { de tipo de empleo. }\end{array}$ \\
\hline $\begin{array}{l}\text { Viviendas } \\
\text { Irrecuperables }\end{array}$ & USACH & Ampliar el concepto de habitabilidad \\
\hline Morbilidad & USACH & $\begin{array}{l}\text { Emplear en concepto de Morbilidad en variable } \\
\text { Enfermedades }\end{array}$ \\
\hline Ítem - Vivienda & USACH & $\begin{array}{l}\text { Generar un nuevo Ítem llamado «Habitat», que } \\
\text { incluya «Vivienda», que además haga referencia a: } \\
\text { Espacios Públicos, Infraestructura comunitaria y } \\
\text { su uso, Sentido de Pertenencia, Allegamiento. }\end{array}$ \\
\hline
\end{tabular}

Análisis de las Mesas de Trabajo

Del trabajo señalado en los puntos anteriores, se decide considerar las sugerencias y observaciones realizadas, con la finalidad de establecer un conjunto de variables que se 
ajuste al concepto de Vulnerabilidad Social Territorial. La tabla 4 recoge nuestras respuestas a las sugerencias y recomendaciones realizadas.

TABLA 4. Análisis de variables. Variables Sugeridas. Fuente: Equipo de investigación del área de Ordenamiento Territorial, Departamento de Ingeniería Geográfica, Universidad de Santiago, año 20I3.

\begin{tabular}{|c|c|c|}
\hline Variables & Mesa de trabajo & Comentarios \\
\hline Integración Social & USACH & $\begin{array}{l}\text { Se integrará un nuevo ITEM que considere los } \\
\text { tópicos de integración social. }\end{array}$ \\
\hline Integración Social & FSP & \\
\hline $\begin{array}{l}\text { Dotación de personal } \\
\text { (Salud) }\end{array}$ & FSP & Se incorporará como una variable en ítem Salud \\
\hline Población Indígena & FSP & $\begin{array}{l}\text { Se incorporará como una variable en ítem Estructura } \\
\text { de Población. Justificando que corresponde a un } \\
\text { grupo sensible de la población a temas de integración } \\
\text { y cambios territoriales. }\end{array}$ \\
\hline \multicolumn{3}{|l|}{ Recomendaciones } \\
\hline Consideraciones & Mesa de trabajo & Comentarios \\
\hline Etnia & USACH & $\begin{array}{l}\text { Fue incorporada dentro del ítem Estructura de } \\
\text { Población }\end{array}$ \\
\hline \% áreas verdes & USACH & $\begin{array}{l}\text { El concepto está incluido en variable } \\
\text { Biodiversidad/paisaje }\end{array}$ \\
\hline $\begin{array}{l}\text { Clasificación por tipo } \\
\text { de trabajo }(*)\end{array}$ & USACH & $\begin{array}{l}\text { Se adecuar \{a la clasificación por la identificación de } \\
\text { estudios obtenidos (disponibilidad de } \\
\text { información) }\end{array}$ \\
\hline Migración & USACH & $\begin{array}{l}\text { Dentro del análisis de migración se considerara lo } \\
\text { recomendado. }\end{array}$ \\
\hline $\begin{array}{l}\text { Viviendas } \\
\text { Irrecuperables }\end{array}$ & USACH & \\
\hline Morbilidad & USACH & Se acogió el cambio del nombre de la variable \\
\hline Ítem - Vivienda & USACH & $\begin{array}{l}\text { Fue acogido el cambio e incluido las variables } \\
\text { propuestas. }\end{array}$ \\
\hline Educación/trabajo & FSP & Se diferenciaran en ítems separados \\
\hline SIMCE & FSP & $\begin{array}{l}\text { Se incluirá una variable de calidad de educación, } \\
\text { como resultados del SIMCE. }\end{array}$ \\
\hline Inacción Juvenil & FSP & Se acogió el cambio del nombre de la variable \\
\hline
\end{tabular}

\section{Etapa III: Variables Seleccionadas}

En este punto se consolida la información obtenida en el proceso de revisión bibliográfica y en las mesas de trabajo, obteniendo un set de variables ajustadas a la naturaleza de este estudio. Cabe destacar que al incorporar la sub-dimensión de Integración Social junto a sus respectivas variables, se agregaron parámetros de valoración subjetivos, los cuales deben ser sometidos a valoración de expertos territoriales del área de estudio. La forma de medición y evaluación de éstos se detallará en el apartado Ponderación de 
Variables / Sub-dimensiones. A continuación se detalla las variables que componen cada sub-dimensión de análisis:

- Hábitat: Condiciones físicas de la vivienda y el entorno.

- Viviendas Irrecuperables: Se refiere a las viviendas que presentan grados de deterioro parcial o total. Una vivienda irrecuperable genera un desmejoramiento desde el punto de vista de habitabilidad y visual.

- Disponibilidad de agua potable: Se consideró como indicador el porcentaje de hogares de la comuna cuya disponibilidad de agua potable califica como aceptable de acuerdo a si posee llave dentro de la vivienda o no.

- Sistema de eliminación de excretas: El indicador considerado en este caso es el porcentaje de hogares de la comuna cuyo sistema de eliminación de excretas califica como aceptable de acuerdo si posee W.C. conectado a alcantarillado o fosa séptica (aceptable), deficitaria en otro caso.

- Materialidad de la vivienda: Este concepto se relaciona con los materiales predominantes en paredes exteriores, cubierta de techo y pisos. Considerado como un activo, asociado a las condiciones con las cuales los habitantes pueden enfrentar diferentes acontecimientos (naturales, sociales).

- Saneamiento: Considera el sistema de distribución de agua potable al interior de la vivienda y el medio de eliminación de excretas, según las ca-tegorías de aceptable y deficitario.

- Hacimiento Habitacional: Se refiere a la proporción de personas por vivienda.

- Infraestructura Comunitaria y su uso: Referido al equipamiento de vivienda y comunitario (Servicios: Financieros, De seguridad, Educacional, Salud, Residuos).

- Espacios Públicos: Espacios destinados al esparcimiento (áreas verdes).

- Accesibilidad-Conectividad: Acceso y disponibilidad a servicios de:

- Accesibilidad al Transporte: Referida al acceso a medios de transporte, así también como al grado de accesibilidad y conexión entre territorios.

- Accesibilidad a los Servicios de Salud: «La ausencia o dificultad en la accesibilidad es un elemento central en el deterioro urbano de las ciudades, en cuanto a la ausencia o insuficiencia en la infraestructura de servicios en general y públicos en particular, son los servicios de salud uno de los tópicos más sensibles dada la demanda existente por parte de la población».

- Accesibilidad a Establecimientos Educacionales: «Es un servicio esencial para alcanzar calidad de vida con estándares aceptables, de lo contrario en este caso particular, dificultades en la accesibilidad y presencia de deterioro puede conllevar niveles de marginalidad en la población» (Romero, 200I). Por lo tanto, la distancia y el tipo de establecimientos son esenciales.

- Accesos a Polos de Desarrollo: El acceso a los servicios de primera necesidad y de ocio es un elemento esencial para determinar los grados de aislamiento. El supuesto metodológico es que a mayor dificultad de acceso a los centros urbanos, que proveen una serie de servicios complementarios a los provistos por el Estado.

- Accesos a Mercado: Acceso a instituciones bancarias.

- Accesos a Servicios Básicos: Acceso a agua y luz. 
- Acceso a telecomunicaciones: Para la determinación de la integración en telecomunicaciones se opta por el indicador de líneas de Internet banda ancha por cantidad de habitantes, debido a que explica, sin sobrevalorar o subvalorar, como son los casos de antenas radioemisoras y de repetición de televisión abierta, que dependen de su localización en función de la cobertura que presentan.

- Suelos: Condiciones de uso de suelo para fines antrópico.

- Valor Promedio del m2 de Terreno: Las características de un terreno inciden directamente en su valor (ubicación, ocupación, uso tipo de superficie y orientación), entre más elementos positivos mayor es su valor. De ésta forma, se restringe la adquisición de éstos.

- Capacidad de uso del suelo: El término «capacidad de uso del suelo» se refiere al potencial de un suelo como recurso para desarrollar diferentes cultivos y formas de agricultura.

- Trabajo: Características poblacionales de las condiciones ocupacionales que posee un territorio.

- Empleabilidad Juvenil: «La inacción juvenil son el desempleo y retraso escolar, los cuales son fenómenos indesmentibles de la desintegración social». «Inacción es considerada precursor de la delincuencia y drogadicción» (Sabatini, Cáceres y Cerda).

- Ingresos del trabajo en el Hogar: son los ingresos por concepto de sueldos y salarios, ganancias provenientes del trabajo independiente, incluido el autosuministro y el valor del consumo de productos agrícolas (aquí se considera el ingreso de la ocupación principal).

- Educación: Características poblacionales de las condiciones educacionales que posee un territorio.

- Escolaridad Promedio: número promedio de años cursados en el sistema educacional, desde primero básico en adelante. Se aplica a las personas de I5 años y más.

- Tasa de analfabetización: Proporción de la población analfabeta respecto a la población total de un territorio determinado.

- Resultados del SIMCE: Resultados del Sistema de medición de calidad de la educación de los establecimientos educacionales.

- Estructura de Población: Composición poblacional que posee un territorio.

- Envejecimiento de Población (porcentaje población tercera edad): Contribuye a generar dependencia demográfica.

- Crecimiento de Población: Proporción de crecimiento de la población de un territorio en el tiempo.

- Migración (interurbana e internacional): Se asocia a competencia laboral, aglomeración, marginalidad. (Dependerá del caso de estudio).

- Hacinamiento Territorial: Relacionada con las viviendas existentes en un territorio (densidad) y la disposición de éstas.

- Etnia: Identificación de la población indígena. 
- Salud: Características de atención e infraestructura que poseen los servicios de salud, así también, las condiciones de salud que poseen la población.

- Indicadores Nutricionales: Grado de desnutrición y/o sobrepeso que presenta la población infantil de un área.

- Infraestructura de Salud (hospitalaria): Tipo de recinto de salud.

- Dotación de Personal: Tipo de profesionales por establecimiento.

- Morbilidad: Complejidad de Enfermedades que presentan los habitantes del área de estudio (asociada a tratamientos, curso de la enfermedad, dependencia).

- Calidad Ambiental: Estado y disponibilidad del recurso, considerando las condiciones de protección.

- Contaminación del suelo: Se refiere a la presencia de restos de sustancias químicas provenientes de actividades antrópicas como, por ejemplo, agroquímicos y/o desechos.

- Calidad del agua referida a:

- Concentración de sólidos: El término concentración de sólidos se refiere a la proporción de sólidos minerales en suspensión en un determinado cuerpo de agua.

- Sustancias tóxicas: Bajo esta denominación se incluyen un conjunto de sustancias tales como desechos industriales, metales pesados y agroquímicas cuyo consumo puede generar reacciones de toxicidad leves o severas en la población humana y animal asentada en la cuenca. Las fuentes de sustancias tóxicas son las actividades industrial, minera y agrícola.

- Compuestos orgánicos, nutrientes y patógenos: La presencia en altas concentraciones de compuestos orgánicos tales como heces, sangre y restos de animales.

- Calidad del aire:

- Concentración de sólidos: El término concentración de sólidos se refiere a la proporción de sólidos minerales en suspensión en un determinado volumen de aire.

- Biodiversidad: El término biodiversidad o diversidad biológica alude a la variabilidad de organismos vivientes y hábitats, así como a los complejos ecológicos de los cuales forman parte. El término biodiversidad incluye la diversidad al interior de las especies, entre especies y de ecosistemas.

- Porcentaje de Superficie Protegida del Deterioro Ambiental: Área silvestres protegidas del Estado y Sitios Prioritarios para la Biodiversidad. El supuesto propuesto es: «A mayor cantidad de área protegidas, menor es el riesgo de deterioro ambiental».

- Riesgos Naturales: Probabilidad de que un territorio y la sociedad que habita en él, se vean afectados por episodios naturales de rango extraordinario.

- Geodinámica: Estudio de los agentes o fuerzas que intervienen en los procesos dinámicos de la Tierra. 
- Inestabilidad de laderas: Es el proceso por el cual las laderas naturales o los taludes construidos por actividades antrópicas pierden estabilidad y se deslizan a través de una superficie de ruptura.

- Fallas activas: El análisis de los rasgos tectónicos permite definir o determinar las fallas y las situaciones de riesgo sísmico que caracterizan las zonas de interés.

- Tsunamis: Zonas de inundación post-Tsunami.

- Integración Socioeconómica: Características socioeconómicas de la población en un territorio.

- Tasa de Detenciones según denuncias de Delitos de Mayor Connotación social (DMCS): Asociada a la percepción de la seguridad ciudadana.

- Línea de Pobreza: Se determina por el nivel de ingreso mínimo necesario por persona para cubrir el costo de una canasta individual para la satis-facción de las necesidades alimentarias y no alimentarias. En la versión 2009 de la CASEN, la «línea de pobreza» se estableció en \$64.I34 per cápita en las zonas urbanas y $\$ 43.242$ per cápita en las rurales.

- Línea de Indigencia: Corresponde al ingreso mínimo necesario por persona para cubrir el costo de una canasta alimentaria. En la versión 2009 de la encuesta CASEN la «línea de indigencia» se determinó en \$32.067 para las zonas urbanas y \$24.7Io para las rurales.

- Activo Financiero: Disponibilidad y procedencia de recursos económicos que posee las autoridades territoriales.

- Fondo Común Municipal (FCM) Autonomía: «Mientras más autonomía tenga una comuna del FCM, menor será su vulnerabilidad social territorial. Se entiende que la dependencia elevada del FCM significa debilidad del municipio y territorio para localizar actividades generadoras de ingresos propios: patentes, permisos de circulación y permisos de edificación de viviendas no exentas de contribuciones».

- Integración Social: La Integración Social es considerada como el proceso por el cual las personas excluidas pueden participar en los procesos y agrupaciones ciudadanas de un determinado territorio. Así también, aspectos que facilitan esta participación. Las variables que componen la integración social:

- Asociatividad: Espacios de encuentro entre los pares y el entorno.

- Organizaciones comunitarias: Nivel de actividad de las organizaciones comunitarias.

- Participación de Gobiernos locales: Iniciativas de las autoridades de involucrar a la población en procesos de planificación local y comunal.

- Participación de Gobiernos regionales: Iniciativas de las autoridades de involucrar a la población en procesos de planificación regional.

- Sector privado: Inversión que genera el sector privado para mejorar el bienestar de la población.

- Sentido de Pertenencia: Identificación de los habitantes con su entorno.

Sintetizando, se tiene el set de variables definitivo en la tabla 5. 
Tabla 5. Variables finales. Fuente: Equipo de investigación del área de Ordenamiento

Territorial, Departamento de Ingeniería Geográfica, Universidad de Santiago, año 20I3.

\begin{tabular}{|c|c|}
\hline Sub-Dimensión & Variable \\
\hline \multirow{8}{*}{ Habitad (Vivienda) } & Viviendas Irrecuperables \\
\hline & Disponibilidad de agua potable \\
\hline & Sistema de eliminación de excretas \\
\hline & Materialidad de la vivienda \\
\hline & Saneamiento \\
\hline & Hacinamiento - Habitacional \\
\hline & Espacios Públicos \\
\hline & Infraestructura comunitaria y su uso \\
\hline \multirow{7}{*}{$\begin{array}{l}\text { Accesibilidad - } \\
\text { Conectividad }\end{array}$} & Accesibilidad al Transporte \\
\hline & Accesibilidad a los Servicios de Salud \\
\hline & Accesibilidad a Establecimientos Educacionales \\
\hline & Acceso a Polos de Desarrollo \\
\hline & Acceso a mercado (instituciones bancarias) \\
\hline & Acceso a telecomunicaciones \\
\hline & Acceso a Servicios Básicos (Agua, Luz) \\
\hline \multirow{3}{*}{ Suelos } & Valor promedio $\mathrm{m} 2$ de terreno \\
\hline & Capacidad de uso del suelo \\
\hline & Propiedades del Suelo \\
\hline \multirow{3}{*}{ Trabajo } & Empleabilidad Juvenil (Inacción Juvenil ) \\
\hline & Ingresos del trabajo en el hogar \\
\hline & Participación Económica (Tasa) \\
\hline \multirow{5}{*}{ Educación } & Promedio de Años de Escolaridad \\
\hline & Escolaridad Promedio \\
\hline & Tasa neta de asistencia \\
\hline & Tasa bruta de asistencia \\
\hline & Resultados SIMCE \\
\hline \multirow{6}{*}{ Estructura de Población } & Envejecimiento de la Población (Porcentaje población 3ra Edad) \\
\hline & Diferencia de Población (Crecimiento) \\
\hline & Habitabilidad del Territorio \\
\hline & Migración (Interurbana e Internacional) \\
\hline & Hacinamiento - Territorial \\
\hline & Etnia \\
\hline \multirow{4}{*}{ Salud } & Indicadores Nutricionales \\
\hline & Dotación de Personal \\
\hline & Infraestructura Hospitalaria \\
\hline & Morbilidad (Enfermedades - Complejidad de Enfermedades) \\
\hline
\end{tabular}




\begin{tabular}{|c|c|}
\hline \multirow{4}{*}{ Calidad Ambiental } & Calidad Agua \\
\hline & Calidad Aire \\
\hline & Calidad de Suelo (Contaminación) \\
\hline & $\begin{array}{l}\text { Porcentaje de Superficie Protegida del Deterioro AmBiental } \\
\text { Biodiversidad - Paisaje }\end{array}$ \\
\hline \multirow{2}{*}{ Riesgos Naturales } & Geodinámica (Inestabilidad, Fallas Activas) \\
\hline & Tsunami (Ubicación Viviendas) \\
\hline \multirow{4}{*}{$\begin{array}{l}\text { Integración } \\
\text { Socioeconómica }\end{array}$} & $\begin{array}{l}\text { Tasa de Detenciones según denuncias de Delitos de Mayor } \\
\text { Connotación Social (DMCS) }\end{array}$ \\
\hline & Integración Social \\
\hline & Línea de la Pobreza \\
\hline & Línea de Indigencia \\
\hline Activo Financiero & Fondo Común Municipal (FCM) - Autonomía \\
\hline \multirow{6}{*}{ Integración Social } & Asociatividad \\
\hline & Organizaciones Comunitarias \\
\hline & Participación de Gobiernos locales \\
\hline & Participación de Gobiernos Regionales \\
\hline & Sector Privado \\
\hline & Sentido de Pertenencia \\
\hline
\end{tabular}

\section{Ponderación de sub-dimensiones y variables}

El proceso consta de cuatro pasos: en el primero se definen los lineamientos (motivos) que formularán la encuesta; luego se construye cada cuestionario (encuesta); el tercer paso es la validación de cada instrumento con el fin de consolidar el cuestionario definitivo; finalmente, se obtiene la encuesta definitiva (figura 3).

\section{Definir Objetivos - Lineamientos}

\section{Construcción de la Encuesta}

\section{Validación del Instrumento}

\section{Encuesta Definitiva}

FIGURA 3. Esquema de ponderaciones de sub-dimensiones y variables. Fuente: Equipo de investigación del área de Ordenamiento Territorial, Departamento de Ingeniería

Geográfica, Universidad de Santiago, año 2013.

\section{Objetivos - Lineamientos}

Se definen los objetivos para los diferentes tópicos que requieren participación de un grupo de actores específicos.

- Objetivo r: Nivel de Sub-Dimensiones 
- Registrar apreciación de los actores territoriales respecto a la agrupación de variables (parámetros).

- Observar el territorio desde el punto de vista de los actores.

- Levantar los ponderadores del Modelo a nivel de Parámetros (sub-di-mensión).

- Objetivo 2: Nivel de Variables

- Levantar los ponderadores de variables que especifican el comportamiento de cada ámbito de la vulnerabilidad (ITEM).

- Registrar la apreciación de expertos afines acerca de las variables específicas.

- Objetivo 3: Integración Social

- Levantar aspectos de difícil apreciación sobre los comportamientos de variables de la integración social.

- Analizar la integración desde el punto de vista de expertos territoriales.

\section{Método de construcción}

Con la finalidad de catastrar cada objetivo, se construyen diferentes tipos de encuestas. A continuación se especifica: tipo, nivel (a quién se aplica) y estructura de las encuestas.

- Tipo de encuesta:

Encuesta I: Nivel de Sub-dimensiones - Actores Territoriales.

Encuesta 2: Nivel Variables - Expertos.

Encuesta 3: Integración Social - Representación del Territorio - Expertos

Territoriales del área de estudio - Valorización de Variables

- Decisión de niveles de encuesta:

Sub-dimensiones: Encuesta aplicada a actores territoriales.

Variables: Encuesta aplicada a expertos territoriales.

Integración Social: Encuesta aplicada a expertos territoriales.

- Estructura de la Encuestas:

Estructura de la Encuesta I - Nivel Sub-Dimensiones: Carta de presentación.

Datos.

Introducción.

Nivel Uno: Conceptos y Definiciones Básicas.

Nivel Dos: Aplicación de Preguntas.

Estructura de la Encuesta 2 - Nivel Variable: Aplicación Directa con los expertos. Mesa de trabajo.

Proceso participativo entre el investigador y colaboradores expertos en los tópicos tratados. 
Estructura de la Encuesta 3 - Integración Social - Valoración de Variables: Carta de presentación.

Datos.

Introducción.

Nivel Uno: Conceptos y Definiciones Básicas.

Nivel Dos: Aplicación de valorización.

\section{Validación (prueba de jueces)}

Para validar cada encuesta se realiza el siguiente proceso.

Paso I: Seleccionar grupo de jueces - Personas que evalúen el instrumento.

Paso 2: Observaciones

Paso 3: Resultados - Análisis.

Paso 4: Retroalimentación

Paso 5: Incorporación de Resultados

Paso 6: Revisión por el grupo de jueces

Paso 7: Si la revisión es aprobada se finaliza el proceso, de lo contrario, se debe volver aplicar el paso 3 con las observaciones emanadas del paso 6.

\section{Encuesta - Definitiva}

Métodos de Valorización de Ponderadores: Como se especificó en puntos anteriores, la valorización de los ponderadores de cada sub-dimensión y variable está compuesta por el juicio de diferentes actores territoriales. La finalidad es contrastar los juicios y valorar los ponderadores. El estudio se divide en dos etapas: Primero se analiza estadísticamente el juicio de los actores, para luego analizar la consistencia lógica de sus respuestas.

- Análisis estadístico: Se estudia: desviación estándar, media, moda. Analizando las tendencias de las respuestas.

- Análisis de consistencia: Para éste análisis se elige el método AHP - Analytic Hierarchy Process, principalmente por sus principios: Construcción de Jerarquías.

- Establecimiento de Prioridades. Consistencia Lógica.

- El método AHP es un instrumento de decisión Multicriterio que traslada la realidad percibida por el individuo a una escala cuantificada, en la que se reflejan las prioridades relativas de los elementos considerados, según Saaty (I980). El método se aplica a la valoración de los ponderadores de variables y sub-dimensiones.

\section{Estudios de Restricciones}

Los tópicos atingentes a este estudio son:

- Aplicación zona de estudio: Se analizará el comportamiento de los principios rectores del modelo propuesto en un área de estudio. Se realizará: Análisis de resultados.

- Contraste metodológico con respecto al modelo de Vulnerabilidad Social Territorial del MDS - GTZ.

- Contraste con diagnóstico del área de estudio realizado por la FSP. 
- Disponibilidad de información: Busca establecer la cantidad de información que requiere el modelo para operar.

- Condiciones Extremas: Pretender explicar el modo de operación cuando se presenta las siguientes condiciones:

- Subvaloración de la ponderación de parámetros y/o variables. Sobrevaloración de la ponderación de parámetros y/o variables.

- Dispersión de la información en la ponderación de parámetros y/o variables (cuando no convergen a un valor).

\section{Resultados}

El modelo propuesto pretende aportar una metodología de análisis territorial enfocada a la vulnerabilidad socio-territorial, entregando una herramienta eficiente para la toma de decisiones en materia de protección social, que permita propender medidas de mejoramiento con el fin de optimizar la gestión del desarrollo y política social en la región del Libertador Bernardo O'Higgins. El estudio de las restricciones se llevará a cabo en el proceso de aplicación a la zona de estudio, la que dependerá también de la disponibilidad de la información y la sub o sobrevalorización de parámetros y variables.

\section{Conclusión}

Al estudiar la Vulnerabilidad Social Territorial por medio de variables y subdimensiones abarcadas en las dimensiones social, físico-ambiental, económica y normativa, el concepto otorga a la representación un carácter integral desde el punto de vista de la disciplina territorial. De igual modo, es preciso conservar dicho carácter en la aplicación de este instrumento, dado que, cualquier malinterpretación u omisión de información jugaría en contra de una adecuada implementación del modelo.

La creación de un set de variables para cada sub-dimensión y dimensión estudiada, brindará a quien aplique el instrumento un punto de partida sustentado en un exhaustivo estudio bibliográfico y de trabajo con expertos del área. Sin embargo, dicho punto debe ser validado por expertos del área específica de estudio. Finalmente, este proceso otorga al modelo un carácter transversal sin excluir la particularidad de cada territorio.

Involucrar participación de los estamentos privados, públicos y de la sociedad civil en la construcción de parámetros y ponderadores, otorga al modelo el carácter de representatividad territorial. Sin embargo, si la muestra no es equitativa entre las partes, dichos ponderadores no serán representativos, por lo tanto, es un aspecto a cuidar en la aplicación. En lo que respecta a la calidad de las respuestas proporcionadas por los encuestados, el indicador de consistencia del modelo brindará el sustento matemático necesario para determinar la información que se empleará.

El instrumento propuesto está compuesto por una serie de variables y subdimensiones, que pueden ser encasilladas en cuatro dimensiones físico-ambiental, social, económico y normativo. Estas variables fueron validadas por una serie de mesas de trabajos y encuestas considerando a profesionales de las distintas áreas de entidades públicas, privadas y sociedad civil, permitiendo transversalidad al momento de elegir el grupo de 
variables que componen el indicador VST. Luego, es de suma importancia que la información con la cual se trabaja sea coherente, para ello es necesario someter toda la información a un proceso de estandarización, proyección y/o normalización. De lo contrario, no tendrían validez los resultados. Eliminar variables y/o sub-dimensiones por falta de información conducirá a valores de la vulnerabilidad VST sesgados, debido a que el concepto de Vulnerabilidad se sustenta en las variables y sub-dimensiones concebidas en el proceso de validación.

Emplear el método AHP en el proceso de construcción de parámetros del modelo es beneficioso desde dos puntos:

I. permite constatar de forma fácil y robusta la información proporcionada en una etapa del modelo;

2. no requiere capital humano altamente capacitado para comprender su aplicación.

Por lo anterior, este proceso resulta ideal para ser transformado en una herramienta de aplicación estatal, al entregar resultados consistentes y no requerir de un exhaustivo proceso de capacitación.

\section{Bibliografía}

ARRIAGAdA LUCO, C. (200I), «Servicios Sociales y Vulnerabilidad en América Latina: conceptos medición e indagación empírica» en Cepal, Seminario Internacional Las diferentes expresiones de la vulnerabilidad social en América Latina y el Caribe, Santiago de Chile, Chile, Comisión Económica para América Latina y El Caribe, Naciones Unidas.

BALDI, G. y GARCÍA, E. (2005), «Calidad de vida y medio ambiente, la psicología ambiental», Universidades, 30, 2005, pp. 9-16.

Busso G. (200I), «Vulnerabilidad Social: Nociones e Implicancias de Políticas para Latinoamérica a inicios del siglo XXI» en CEPAL, Seminario Internacional Las diferentes expresiones de la vulnerabilidad social en América Latina y el Caribe, Santiago de Chile, Chile, Comisión Económica para América Latina y El Caribe, Naciones Unidas.

BUSTAMANTE, J. (2000), Un marco de referencia acerca de la vulnerabilidad de los migrantes como sujetos de los derechos humanos, documento presentado al Taller sobre mejores prácticas en materia de migración, Comisión Económica para América Latina.

CARO, E. (2003), «La Vulnerabilidad Social como enfoque de análisis de la política de asistencia social para la población adulta mayor en México» en Simposio Viejos y Viejas Participación, Ciudadanía e Inclusión Social, 5I Congreso Internacional de Americanistas, Santiago, Chile.

CEPAL (1997), Panorama Social de América Latina, Santiago de Chile, Comisión Económica para América Latina.

CEPAL (2006), «La protección social de cara al futuro: acceso, financiamiento y solidaridad», XXXI periodo de sesiones, Comisión Económica para América Latina y el Caribe, Montevideo, Uruguay, CEPAL.

CEPAL (20I0), Panorama Social de América Latina, Chile, Comisión Económica para América Latina y El Caribe, 262 páginas, http://www.cepal.org/es/publicacio nes/I236-panorama-socialamerica-latina-20I0 (Fecha de consulta: 2/5/20I7).

CUTTER, S. BorufF, B. y SHIRLEY, L. (2003), «Social vulnerability to environmental hazard», Social Science Quarterly, 84/2, pp. 242-26I.

Cutter, S. L., Barnes, L., Berry, M., Burton, M., Evans, E., TAte, E. y WebB, J. (2008), «A placebased model for understanding community resilience to natural disasters». Global Environmental Change, 18/4, pp. 598-606. 
FundACIÓN SUPERACIÓN DE LA POBREZA (2009), «Umbrales sociales para Chile: hacia una futura política social», http://www.superacionpobreza.cl/wp-content/uploads/2014/02/UMBRALES _SOCIALES.pdf (Fecha de consulta: 2/5/2017).

Gacitúa, E., Sojo, C. y Shelton, D. (2000), «Exclusión Social y Reducción de La Pobreza en América Latina y el Caribe». FLACSO, Costa Rica, Banco Mundial, 3I2 páginas.

KAZTMAN, R. (2003), «Notas sobre la medición de la Vulnerabilidad Social», http://www. cepal.org/deype/mecovi/docs/taller5/24.pdf (Fecha de consulta: 2/5/20I7).

MIDEPLAN (2004), «Conceptos Fundamentales: Sist. de Protección Social - Chile Solidario», Chile, Gobierno de Chile, Min. Planificación y Cooperación. 52 pág. http://www.ministeriodesarrollosocial.gob.cl/btca/txtcompleto/chs/ centrodoc_I7o.pdf (fecha de consulta: 2/5/20I7).

MINVU (2006), «Medición del Déficit Habitacional», http://www.minvu.cl/opensite_200704III64536.aspx (fecha de consulta: 2/5/20I7).

Perles Roselló, M.J. (20IO), «Apuntes para la evaluación de la vulnerabilidad social frente al riesgo», Baética, 32, pp. 67-87, ISSN: 0212-5099.

PiZArro, R. (200I), La Vulnerabilidad Social y sus Desafíos: una mirada desde América Latina, Santiago de Chile, CEPAL, 72 pp., http://repositorio.cepal.org/bitstream/handle/II362/4762/ Soio2ıi6_es.pdf (fecha de consulta: 2/5/20I7).

PNUD (2000): Más Sociedad para Gobernar el Futuro, Programa de Naciones Unidas para el Desarrollo.

Romero, CARlos (200I), «Ciudad, cultura y turismo: calidad y autenticidad», Boletín del Instituto Andaluz del Patrimonio Histórico, 36.

SAATY, T. L. (I980), The Analytic Hierarchy Process, New York, McGraw Hill.

SABATINi, F., CÁCERES, G. y CERDA, J. (200I), «Segregación residencial en las principales ciudades chilenas: Tendencias de las tres últimas décadas y posibles cursos de acción», EURE, 27/82.

Vignolo, C. (2004), «Desencadenando la Innovación en la Empresa del Tercer Milenio», Revista Trend Management, 6, I7 pp. 